\title{
Estoque e frações de carbono orgânico e fertilidade de solo sob floresta, agricultura e pecuária
}

\author{
Marihus Altoé Baldotto ${ }^{1}$, Erli Maciel Vieira ${ }^{2}$, Dálisson de Oliveira Souza ${ }^{2}$, Lílian Estrela Borges Baldotto ${ }^{1}$ \\ http://dx.doi.org/10.1590/0034-737X201562030010
}

\section{RESUMO}

O entendimento dos fenômenos de trocas de carbono entre solo, água e atmosfera é fundamental para o manejo e a conservação de ecossistemas naturais e agrários. Os objetivos deste trabalho foram determinar os estoques de carbono orgânico, as suas frações e a fertilidade do solo sob os manejos florestal (mata remanescente), integrando agricultura e florestas (eucalipto e cutieira) e em monoculturas agrícola (sob pivô central) e pastoril (braquiária), na Bacia do Rio Paraopeba, em Florestal-MG, visando a propor indicadores para a avaliação de manejo e conservação do solo. As amostras de solo foram coletadas em remanescente de mata nativa (Mata), em área agrícola cultivada sob irrigação de pivô central (Pivô) e em áreas de cultivo de Corymbia citriodora (Corymbia citriodora (Hook.) K.D. Hill \& L.A.S. Johnson) (Eucalipto), Joannesia princeps Vell. (Cutieira) e Brachiaria decumbens Stapf (Pasto), nas profundidades de 0 a 20 e 20 a $40 \mathrm{~cm}$. Os resultados indicaram que as estimativas dos estoques e de estabilidade do carbono orgânico aumentaram com a fertilidade do solo. Os sistemas florestais, principalmente a Mata, acompanhada pelos sistemas de manejo do solo integrando florestas à pastagem, em comparação com o cultivo contínuo ou a monocultura de pastagem, apresentaram estoques de carbono maiores, mais estáveis e menos solúveis, com formas mais aromáticas e hidrofóbicas (maior relação AH/AF), indicando menor potencial de lixiviação de carbono para o sistema aquático adjacente.

Palavras-chave: manejo e conservação do solo, química do solo, matéria orgânica do solo, ciências ambientais.

\section{ABSTRACT}

\section{Organic carbon stocks and fractions and soil fertility under forest, agriculture and livestock}

Understanding the phenomena of carbon exchange between soils, waters and atmosphere is critical to the management and conservation of agrarian and natural ecosystems. The objective of this study was to determine the organic carbon stocks and their fractions, and the soil fertility under different forest management practices, agricultural and pastoral, on the campus of Florestal, Federal University of Viçosa, aiming to propose indicators for the assessment of soil management and conservation. Organic carbon stocks and fractions were determined and chemical analysis was performed for soil samples collected at depths of 0 to 20 and 20 to $40 \mathrm{~cm}$ under native forest remnants (Forest), farmlands under center pivot irrigation (Pivot) and areas of cultivation of Corymbia citriodora (Corymbia citriodora (Hook.) K.D. Hill \& L.A.S. Johnson) (Eucalyptus), Joannesia princeps Vell. (Cutieira) and Brachiaria decumbens Stapf (Pasture). The results indicated that the organic carbon stokes and stability increased with the soil fertility. The forest systems, mainly the native forest remnants together with management systems integrating forests with livestock, compared with continuous cropping or monoculture pasture had larger, more stables and less soluble carbon stocks, with more aromatic and hydrophobic forms (greater HA/FA ratio), indicating lower leaching potential of carbon to the adjacent aquatic system.

Key words: management and soil conservation, soil chemistry, soil organic matter, environmental sciences.

\footnotetext{
Submetido em 25/03/2013 e aprovado em 27/05/2015.

${ }^{1}$ Universidade Federal de Viçosa, Florestal, Minas Gerais, Brasil. marihus@ufv.br; lilian.estrela@ufv.br

${ }^{2}$ Universidade Federal de Viçosa, Florestal, Minas Gerais, Brasil. erli.m.vieira@ gmail.com; dalissondos@ hotmail.com

*Autor para correspondência: marihus@ufv.br
} 


\section{INTRODUÇÃO}

A produção agropecuária apresenta-se em crescimento em todo o mundo, graças a seu potencial de geração de renda e emprego, além dos seus benefícios ambientais, advindos dos aumentos contínuos de produtividade. Dentre os fatores de produção agropecuária, torna-se fundamental a preocupação com o manejo e a conservação do solo. O foco atual da pesquisa pedológica é a necessidade constante de aperfeiçoamento do manejo e conservação de solo em atividades agropecuárias no país, como a erosão e a diminuição do estoque de carbono orgânico do solo. Para apresentar soluções a estes problemas, destaca-se a busca contínua da ciência pelos sistemas de manejo integrados, que promovem uma agricultura tropical no Brasil, já exemplar e em contínuo aperfeiçoamento (Baldotto et al., 2010).

Desde o início da colonização do Brasil até a agricultura atual, observou-se a transição entre sistemas itinerantes, o desenvolvimento de tecnologias para correção da acidez do solo e o uso de fertilizantes, a calibração de doses recomendáveis de nutrientes, em consonância com os materiais genéticos avançados que surgiram e foram incluídos no manejo integrado, o qual considerou também o monitoramento e o manejo de fitossanidade, as plantas daninhas, a irrigação, a colheita e a comercialização (economia), etc. Com base na disponibilidade crescente de tecnologias, novas fronteiras agrícolas foram incorporadas e as produtividades vêm alcançando patamares recordes, contribuindo para a otimização das áreas agrícolas atuais, sem pressionar novas fronteiras e, ainda, sendo um dos principais pilares da economia nacional. A agropecuária brasileira tornou-se altamente competitiva, mesmo considerando-se as políticas de subsídio dos concorrentes internacionais. Nesse mesmo sentido, foi monitorada a qualidade dos sistemas agrícolas e, entre outros problemas, verificaram-se a erosão e a diminuição do estoque de carbono orgânico do solo, bem como de seu corolário nas propriedades químicas, físicas e biológicas dos agroecossistemas. Como solução e como primeiro salto tecnológico, foi desenvolvido o Sistema Plantio Direto, cujos benefícios já são detalhadamente comprovados. No mesmo sentido da agropecuária, o setor florestal aparece como gerador de alta tecnologia e alavanca a economia brasileira, pois a madeira plantada é fundamental em nossa civilização. Em paralelo, o status quo demanda que os agroecossistemas busquem semelhanças com os ecossistemas originais na fronteira agrícola brasileira, ou seja, é necessário reintroduzir a floresta nos sistemas de manejo. Por essas razões, além de diversificar as atividades e a alocação sazonal dos meios de produção, distribuindo melhor a força de trabalho, a integração entre lavoura, pecuária e floresta pode ser a evolução dos sistemas de manejo agropecuário para este novo século (Baldotto et al., 2010), indo ao encontro dos mecanismos de desenvolvimento limpo, sobretudo de mitigação do efeito estufa (IPCC, 2013).

Assim, as atuais evidências da contribuição do $\mathrm{CO}_{2}$ atmosférico para o aquecimento global têm aumentado o interesse pelo carbono orgânico do solo, por ser um dos principais compartimentos do ciclo terrestre deste elemento (Eswaran et al., 2001; Cerri et al., 2003; Lal, 2004; Carvalho et al., 2010; Baldotto et al., 2010). A maior parte do carbono orgânico fixado pelas plantas é capturada na fotossíntese, retornando para a atmosfera na forma de $\mathrm{CO}_{2}$, por decomposição dos resíduos vegetais (Stevenson, 1994; Sposito, 2008). O desmatamento e as práticas agrícolas intensivas, como as arações e gradagens, aumentam a transferência de carbono da biomassa para atmosfera (Fang \& Moncrieff, 2001; IPCC, 2001; Lal, 2004).

A matéria orgânica do solo é um indicador sensível de alterações do solo, decorrentes do seu uso e ocupação. O estoque, as formas de carbono orgânico e as características químicas do solo estão estreitamente relacionados e variam de acordo com as práticas de manejo (Derpsch, 1997; Dias \& Griffith, 1998; Eswaran et al., 2001; Reis \& Rodella, 2002; Cerri et al., 2003; Lal, 2004; Carvalho et al., 2010; Baldotto et al., 2010).

De forma geral, as principais limitações dos solos tropicais, sobretudo da maioria dos Latossolos, que são o insuficiente desenvolvimento de cargas elétricas negativas (baixa CTC), a elevada fixação de fosfatos, a baixa disponibilidade de nutrientes (bases e P) e as altas concentrações de íons alumínios tóxicos $\left(\mathrm{Al}^{3+}\right)$, podem ser minimizados com a preservação e o incremento dos teores de carbono orgânico do solo, uma vez que a matéria orgânica apresenta grupos funcionais eletricamente carregados, que aumentam a CTC e diminuem a adsorção específica de $\mathrm{P}$, disponibiliza nutrientes e tem a capacidade de complexar íons alumínio, reduzindo sua toxicidade.

Nesse sentido, como os Latossolos distribuem-se em praticamente todo o território nacional, sendo a classe de solos mais representativa do Brasil (Coelho, 2002; Embrapa, 2006), ocupando cerca de $60 \%$ do território (Correia, 2004) e são solos de grande potencial para agricultura, espera-se que preservar os estoques de carbono seja uma forma eficiente de manter importantes propriedades dos Latossolos. Vários estudos têm indicado que a integração de lavoura e pecuária com florestas tem possibilitado a recuperação dos teores de matéria orgânica.

Os objetivos deste trabalho foram determinar os estoques de carbono orgânico e as suas frações e a fertili- 
dade do solo sob os manejos florestal (mata remanescente), integrando agricultura e florestas (eucalipto e cutieira) e as monoculturas agrícola (sob pivô central) e pastoril (braquiária), na Bacia do Rio Paraopeba, em Florestal-MG, visando a propor indicadores para a avaliação de manejo e da conservação do solo.

\section{MATERIAL E MÉTODOS}

Este trabalho foi realizado em Florestal-MG, estudando-se um complexo lacustre típico da região, conhecido como "lagoas da CEDAF". A área pertence à Universidade Federal de Viçosa - Campus Florestal (UFVCAF), localizada nas coordenadas de 1952'16,3'S e $44^{\circ} 25^{\prime} 26,1$ ' $\mathrm{W}$, a uma altitude de aproximadamente 750 metros. A área localiza-se na bacia do Rio Paraopeba e está na abrangência de duas sub-bacias, as do Ribeirão das Lages e do Ribeirão do Ouro (CIBAPAR, 2013).

Foram amostradas cinco áreas adjacentes no entorno do sistema hídrico, compondo remanescentes florestais (matas nativas), integração de florestas e pecuária (eucaliptos e cutieiras) e monocultura (agricultura sob pivô central e pastagem). A área foi denominada "Mata", quando constituída por solos sob a cobertura de remanescentes de transição Cerrado-Mata Atlântica; "Eucalipto", "Cutieira" e "Pasto" representaram solos sob matas de Eucalyptus ssp., Joannesia princeps e Brachiaria decumbens, respectivamente. Uma área agrícola, sob pivô central e manejada há aproximadamente duas décadas, cuja referência no texto será "Pivô", em sistema convencional, com aração e gradagens. O manejo com Eucalipto e Cutieira tem aproximadamente 25 anos. As pastagens plantadas de braquiária também foram implantadas em épocas semelhantes.

$\mathrm{O}$ entorno das unidades de amostragem apresenta relevo predominantemente suave ondulado e o relevo regional ondulado. $\mathrm{O}$ solo amostrado pertence à classe Latossolo Amarelo distrófico argissólico, conforme o Sistema Brasileiro de Classificação de Solos (Embrapa, 2006). Foram coletadas amostras compostas de solo nas camadas de 0 a 20 e de 20 a $40 \mathrm{~cm}$, conforme recomendações de Embrapa (1997; 2006). As amostras de solo foram destorroadas, peneiradas em malhas de $2 \mathrm{~mm}$ e secas em bancada à sombra, para a obtenção da terra fina seca ao ar (TFSA), usadas nas análises química e física do solo (Embrapa, 1997).

De posse dos teores de carbono orgânico e da densidade do solo, foram calculados os Estoques de Carbono por meio da equação:

$$
\mathrm{EC}=\mathrm{C} \times \mathrm{p} \times \mathrm{DS} \times 10
$$

na qual, $\mathrm{C}$ é o teor de carbono orgânico em $\mathrm{g} \mathrm{kg}^{-1}$, p é a camada amostrada em m, DS é a densidade do solo em
$\mathrm{Mg} \mathrm{m}^{-3}$, conforme Baldotto et al, 2010. O EC é apresentado, conforme o Sistema Internacional de Unidades, megagrama por hectare $(\mathrm{Mg} / \mathrm{ha})$, sendo $\mathrm{Mg}$ equivalente à tonelada.

O fracionamento, a extração e a purificação do carbono orgânico do solo seguiram as recomendações da International Humic Substances Society (IHSS, 2013), descritas em Thurman \& Malcolm (1981). Inicialmente, as amostras foram tratadas com $\mathrm{H}_{3} \mathrm{PO}_{4} 2 \mathrm{~mol} \mathrm{~L}^{-1}$ (agitação, em tubos de centrífuga, de $20 \mathrm{~g}$ de amostra: 200 $\mathrm{mL}$ de solução por 4 horas) para a extração da fração ácidos fúlvidos livres (AFL) e da matéria orgânica leve (MOL). Após essa agitação, foi realizada a centrifugação (Centrífuga HERMLE-Z382K), a 5.000 g, por 30 minutos. A densidade ótica do extrato sobrenadante foi medida em espectrofotometria de absorção molecular, em equipamento Hitashi - UV-Vis 2000, nos comprimentos de onda de 250 e $465 \mathrm{~nm}$. Para a extração de substâncias orgânicas alcalino-solúveis, o precipitado do extrato obtido na etapa anterior foi ajustado para $\mathrm{pH} 7 \mathrm{e}$, em seguida, adicionaram-se ao tubo de centrífuga 200 $\mathrm{mL} \mathrm{NaOH} \mathrm{0,5} \mathrm{mol} \mathrm{L}^{-1}$. Esse sistema, a $\mathrm{pH} \sim 13$, foi agitado horizontalmente, por 24 horas. As amostras foram centrifugadas e determinadas as densidades óticas dos sobrenadantes e, também, seus teores de carbono orgânico, contendo as substâncias húmicas (SH) e os precipitados residuais (Huminas). Parte dos sobrenadantes da operação anterior foi acidificada, até pH 1,5, com ácido sulfúrico e, a seguir, centrifugada, para a separação das $\mathrm{SH}$ em ácidos húmicos ( $\mathrm{AH})$, no precipitado, e de ácidos fúlvicos (AF), no sobrenadante.

Estimaram-se a média e o desvio padrão da média para cada variável experimental e foi também realizada a análise multivariada dos dados, obtendo-se o teste de agrupamento, usando-se a distância euclidiana, uma medida da dissimilaridade entre as amostras, determinada a partir das variáveis de um conjunto amostral. O resultado final da análise multivariada dos dados foi um dendrograma, no qual, quanto mais distantes as amostras, menor é a sua semelhança (Cruz, 2006). Para as análises estatísticas, foi usado o Programa SAEG 9.0.

\section{RESULTADOS E DISCUSSÃO}

Os resultados das análises químicas, de densidade e de estoques de carbono do solo, em ambas as profundidades de amostragem, encontram-se nas Tabelas 1 e 2 respectivamente. Os resultados foram interpretados a partir das recomendações para o Estado de Minas Gerais, apresentadas por Alvarez V. et al. (1999).

A matéria orgânica do solo, na camada 0 a $20 \mathrm{~cm}$, apresentou teores médios para os sistemas Mata, Eucalipto, Cutieira e Pasto. Para o solo sob Pivô, o teor 
70 Tabela 1. Características químicas dos solos no entorno dos Lagos do Campus de Florestal da UFV, na Bacia do Rio Paraopeba, sob distintas coberturas adjacentes

\begin{tabular}{|c|c|c|c|c|c|c|c|c|c|c|c|c|c|c|}
\hline \multirow[t]{3}{*}{ Sistema $^{(1)}$} & \multicolumn{14}{|c|}{ Características Químicas $^{(2)}$} \\
\hline & \multirow{2}{*}{$\begin{array}{c}\text { MOS } \\
\text { dag kg }\end{array}$} & \multirow[t]{2}{*}{ pH } & $\mathbf{P}$ & $\mathbf{K}$ & $\mathbf{C a}^{+2}$ & $\mathrm{Mg}^{+2}$ & $\mathbf{A l}^{+3}$ & $\mathbf{H}+\mathbf{A l}$ & SB & $\mathbf{t}$ & $\mathbf{T}$ & $\mathbf{V}$ & $\mathbf{m}$ & P-Rem \\
\hline & & & \multicolumn{2}{|c|}{$\mathrm{mg} \mathrm{dm}{ }^{-3}$} & \multicolumn{7}{|c|}{$\mathrm{cmol}_{\mathrm{c}} \mathrm{dm}^{-3}$} & \multicolumn{2}{|c|}{$\%$} & $\mathrm{mg} \mathrm{L}^{-1}$ \\
\hline & \multicolumn{14}{|c|}{ Camada 0 a $20 \mathrm{~cm}$} \\
\hline Mata & $2,97 \pm 0,09$ & $5,25 \pm 0,04$ & $5,65 \pm 0,35$ & $94,50 \pm 1,68$ & $1,56 \pm 0,08$ & $0,73 \pm 0,03$ & $0,30 \pm 0,28$ & $5,40 \pm 0,85$ & $2,53 \pm 0,15$ & $2,82 \pm 0,13$ & $7,93 \pm 1,00$ & $32,00 \pm 2,12$ & $10,25 \pm 9,26$ & $55,50 \pm 0,99$ \\
\hline Eucalipto & $3,05 \pm 0,21$ & $3,94 \pm 0,02$ & $2,15 \pm 0,21$ & $15,00 \pm 1,41$ & $0,20 \pm 0,03$ & $0,16 \pm 0,01$ & $1,71 \pm 0,07$ & $7,45 \pm 0,35$ & $0,40 \pm 0,04$ & $2,11 \pm 0,11$ & $7,85 \pm 0,31$ & $5,10 \pm 0,71$ & $81,10 \pm 0,99$ & $33,80 \pm 2,97$ \\
\hline Cutieira & $2,84 \pm 0,09$ & $4,91 \pm 0,06$ & $2,80 \pm 0,14$ & $47,50 \pm 1,61$ & $0,52 \pm 0,08$ & $0,27 \pm 0,04$ & $0,83 \pm 0,07$ & $6,05 \pm 0,07$ & $0,91 \pm 0,09$ & $1,74 \pm 0,16$ & $6,96 \pm 0,16$ & $13,00 \pm 0,99$ & $47,85 \pm 0,35$ & $39,25 \pm 0,49$ \\
\hline Pivô & $1,78 \pm 0,83$ & $4,97 \pm 0,12$ & $6,05 \pm 5,87$ & $14,50 \pm 3,54$ & $0,74 \pm 0,11$ & $0,16 \pm 0,06$ & $0,73 \pm 0,48$ & $4,45 \pm 2,19$ & $0,94 \pm 0,18$ & $1,67 \pm 0,66$ & $5,39 \pm 2,37$ & $18,40 \pm 4,81$ & $41,35 \pm 12,52$ & $39,75 \pm 6,72$ \\
\hline \multirow[t]{2}{*}{ Pasto } & $2,24 \pm 0,00$ & $5,80 \pm 0,00$ & $13,20 \pm 0,57$ & $27,00 \pm 2,28$ & $1,22 \pm 0,01$ & $0,66 \pm 0,01$ & $0,00 \pm 0,00$ & $2,95 \pm 0,35$ & $1,95 \pm 0,09$ & $1,95 \pm 0,09$ & $4,90 \pm 0,26$ & $39,85 \pm 4,03$ & $0,00 \pm 0,00$ & $54,90 \pm 0,99$ \\
\hline & \multicolumn{14}{|c|}{ Camada 20 a $40 \mathrm{~cm}$} \\
\hline Mata & $2,44 \pm 0,09$ & $4,72 \pm 0,02$ & $4,85 \pm 0,92$ & $40,50 \pm 2,12$ & $0,23 \pm 0,01$ & $0,36 \pm 0,03$ & $0,93 \pm 0,21$ & $5,90 \pm 0,57$ & $0,69 \pm 0,04$ & $1,62 \pm 0,25$ & $6,59 \pm 0,61$ & $10,50 \pm 0,28$ & $57,00 \pm 3,96$ & $42,95 \pm 1,77$ \\
\hline Eucalipto & $2,04 \pm 0,28$ & $4,02 \pm 0,01$ & $1,50 \pm 0,00$ & $11,00 \pm 1,41$ & $0,00 \pm 0,00$ & $0,09 \pm 0,01$ & $1,81 \pm 0,61$ & $6,65 \pm 0,78$ & $0,12 \pm 0,01$ & $1,92 \pm 0,06$ & $6,77 \pm 0,78$ & $1,70 \pm 0,14$ & $94,00 \pm 0,57$ & $27,35 \pm 0,35$ \\
\hline Cutieira & $1,26 \pm 0,09$ & $4,75 \pm 0,02$ & $1,40 \pm 0,14$ & $7,50 \pm 2,12$ & $0,07 \pm 0,02$ & $0,08 \pm 0,00$ & $1,22 \pm 0,07$ & $4,20 \pm 0,71$ & $0,17 \pm 0,02$ & $1,39 \pm 0,05$ & $4,37 \pm 0,73$ & $3,80 \pm 0,14$ & $88,05 \pm 1,91$ & $35,00 \pm 1,84$ \\
\hline Pivô & $1,98 \pm 0,19$ & $4,60 \pm 0,18$ & $6,20 \pm 0,14$ & $12,00 \pm 0,00$ & $0,36 \pm 0,02$ & $0,09 \pm 0,01$ & $1,32 \pm 0,07$ & $7,50 \pm 2,83$ & $0,48 \pm 0,04$ & $1,80 \pm 0,04$ & $7,98 \pm 2,79$ & $6,40 \pm 2,69$ & $73,55 \pm 2,47$ & $31,15 \pm 0,64$ \\
\hline Pasto & $1,12 \pm 0,08$ & $5,11 \pm 0,04$ & $2,00 \pm 0,00$ & $11,50 \pm 0,71$ & $0,76 \pm 0,01$ & $0,14 \pm 0,01$ & $0,54 \pm 0,07$ & $3,30 \pm 0,28$ & $0,92 \pm 0,00$ & $1,46 \pm 0,07$ & $4,22 \pm 0,28$ & $21,85 \pm 1,48$ & $36,95 \pm 3,04$ & $43,70 \pm 0,57$ \\
\hline
\end{tabular}

(1) Sistema: Mata = cobertura remanescente de transição Mata Atlântica-Cerrado; Eucalipto, Cutieira e Pastagem = cobertura das espécies Eucalyptus citriodora, Joannesia princeps e Brachiaria decumbens; Pivô = área agrícola sob pivô central desativado e manejada atualmente sob pastagem com predomínio de $B$. decumbens; ${ }^{(2)}$ Características Químicas: MOS = matéria orgânica do solo $($ Walkey \& Black); $\mathrm{pH}=$ relação solo:água na relação 1:2,5; Pe K = extrator Mehlich-1; $\mathrm{Ca}^{2+}, \mathrm{Mg}^{2+} \mathrm{e} \mathrm{Al}{ }^{3+}=$ extrator $\mathrm{KCl} 1 \mathrm{~mol} \mathrm{~L}^{-1} ; \mathrm{H}+\mathrm{Al}=$ acetato de cálcio $0,5 \mathrm{~mol} \mathrm{~L}^{-1}, \mathrm{pH} 7,0 ; \mathrm{SB}=\mathrm{K}^{+}+\mathrm{Ca}^{2+}+\mathrm{Mg}^{2+}+\mathrm{Al}{ }^{3+} ; \mathrm{t}=\mathrm{SB}+\mathrm{Al}{ }^{3+} ; \mathrm{T}=\mathrm{SB}+(\mathrm{H}+\mathrm{Al}) ; \mathrm{V}=(\mathrm{SB} / \mathrm{T}) \times 100 ; \mathrm{m}=\left(\mathrm{Al} l^{3+} / \mathrm{t}\right) \times 100 ;$ $\mathrm{P}-\mathrm{Rem}$ = fósforo remanescente, concentração de fósforo na solução de equilíbrio após agitar durante uma hora a TFSA com solução $\mathrm{CaCl}_{2} 10 \mathrm{mmol} \mathrm{L}^{-1}$, contendo $60 \mathrm{mg} \mathrm{L}{ }^{-1}$ de $\mathrm{P}$, na relação 1: 10; As médias estão acompanhadas do seu desvio padrão. 
de matéria orgânica foi baixo. Na camada 20 a $40 \mathrm{~cm}$, a matéria orgânica determinada foi baixa para Pivô, Pasto e Cutieira e média para Eucalipto e Mata.

Os valores de $\mathrm{pH}$ apresentaram-se médios nos sistemas Mata e Pasto, na camada de 0 a $20 \mathrm{~cm}$ do solo, e a acidez ativa foi classificada como elevada na Cutieira e no Pivô. O solo sob Pasto apresentou pH médio, enquanto Mata, Cutieira e Pivô revelaram valores elevados. $\mathrm{O}$ $\mathrm{pH}$ do solos sob Eucalipto, em ambas as camadas, foi muito baixo.

Com relação à acidez trocável, foi alta para Eucalipto, média para Cutieira e Pivô e muito baixa para Mata e Pasto, sendo que o $\mathrm{Al}^{3+}$ foi igual a zero neste último sistema, na profundidade 0 a $20 \mathrm{~cm}$. Já na camada subsequente, Mata e Pasto apresentaram teores médios de acidez trocável, e Eucalipto, Cutieira e Pivô revelaram altos teores de $\mathrm{Al}^{3+}$.

A acidez potencial também se apresentou em apenas duas classes, média e alta. Na camada superficial, $\mathrm{H}+\mathrm{Al}$ seguiu a ordem Eucalipto > Cutieira > Mata > Pivô > Pasto e, na camada de 20 a $40 \mathrm{~cm}$, Pivô > Eucalipto > Mata $>$ Cutieira > Pasto.

Considerando-se o teor de fósforo, observaram-se magnitudes muito baixas para todos os sistemas, em ambas as profundidades de amostragem, exceto para a camada superficial da pastagem.

Em todos os sistemas avaliados, os teores de $\mathrm{K}, \mathrm{Ca}^{2+}$ e $\mathrm{Mg}^{2+}$ variaram de muito baixos a médios, com exceção do teor de K, no solo superficial sob Mata, o qual foi classificado como bom. Contudo, não foi suficiente para alterar o resultado da soma de bases, que permaneceu entre as classes muito baixa a média. Mesmo assim, a ordem dos valores foi: Mata > Pasto > Pivô, Cutieira, Eucalipto, na camada 0 a $20 \mathrm{~cm}$, e Pasto > Mata > Pivô > Cutieira $>$ Eucalipto, na profundidade 20 a $40 \mathrm{~cm}$.
A capacidade de troca de cátions (CTC), tanto efetiva, como total, variou de baixa a média, para todos os sistemas e em ambos os horizontes. A Mata mostrou valores médios de CTC nas duas camadas estudadas.

Relacionando-se a soma de bases com a CTC dos solos, observou-se que a saturação por bases, na camada superficial, apresentou-se baixa para Mata e muito baixa nos sistemas Cutieira, Pivô e Eucalipto. Já na amostragem em subsuperfície, também foi muito baixa em todos os sistemas, à exceção do solo sob Pasto, no qual foi baixa.

O fracionamento químico dos estoques de carbono totais dos sistemas estudados revelou diferenças químicoestruturais em sua composição (Tabela 3).

Na camada superficial, as concentrações da fração MOL seguiram a ordem Pasto Pivô < Cutieira < Eucalipto Mata, sendo a amplitude dos valores de 1,19 a $2,67 \mathrm{~g} \mathrm{~kg}^{-1}$. A mesma ordem de MOL aconteceu na camada subsuperficial, porém variando de 0,50 a 0,87 $\mathrm{g} \mathrm{kg}^{-1}$.

Os valores de AFL na camada 0 a $20 \mathrm{~cm}$ foram mais elevados nos sistemas Eucalipto e Mata, seguidos pelo Pasto, Pivô e Cutieira. Na camada 20 a 40 cm, o comportamento da fração AFL também foi maior na Mata e no Eucalipto, acompanhados, na sequência por Pastagem, Cutieira e Pivô. As amplitudes dos valores de AFL foram de 1,23 a $2,47 \mathrm{~g} \mathrm{~kg}^{-1}$ na camada superficial e de 0,65 a $2,35 \mathrm{~g} \mathrm{~kg}^{-1}$ na camada subsuperficial.

A fração AF variou menos na camada 0 a $20 \mathrm{~cm}$, de 1,52 a 2,13 $\mathrm{g} \mathrm{kg}^{-1}$, mantendo-se mais elevada nas áreas de Mata e Eucalipto, seguidas por Cutieira, Pastagem e Pivô. Já na camada 20 a $40 \mathrm{~cm}$, foi verificado comportamento menos homogêneo e os valores de AF variaram de 0,59 a $1,60 \mathrm{~g} \mathrm{~kg}^{-1}$.

A concentração de AH seguiu a ordem Pivô < Pastagem $\sim$ Cutieira $<$ Eucalipto $<$ Mata na camada superfici-

Tabela 2. Teor de carbono orgânico, densidade do solo e estoque de carbono do solo sob diferentes coberturas vegetais na Bacia do Rio Paraopeba

\begin{tabular}{|c|c|c|c|}
\hline Sistema & $\begin{array}{c}\mathrm{C} \\
\mathrm{g} \mathrm{kg}^{-1}\end{array}$ & $\begin{array}{c}\text { DS } \\
\mathrm{Mg} \mathrm{m}^{-3}\end{array}$ & $\begin{array}{c}\text { EC } \\
\text { Mg ha }^{-1}\end{array}$ \\
\hline \multicolumn{4}{|c|}{ Camada 0 a $20 \mathrm{~cm}$} \\
\hline Mata & $1,72 \pm 0,05$ & $1,00 \pm 0,02$ & $34,24 \pm 1,79$ \\
\hline Eucalipto & $1,77 \pm 0,12$ & $1,07 \pm 0,01$ & $37,81 \pm 3,04$ \\
\hline Cutieira & $1,64 \pm 0,05$ & $1,07 \pm 0,01$ & $35,18 \pm 0,68$ \\
\hline Pivô & $1,03 \pm 0,48$ & $1,10 \pm 0,01$ & $22,78 \pm 1,09$ \\
\hline Pasto & $1,30 \pm 0,00$ & $1,01 \pm 0,03$ & $26,25 \pm 0,73$ \\
\hline \multicolumn{4}{|c|}{ Camada 20 a $40 \mathrm{~cm}$} \\
\hline Mata & $1,41 \pm 0,05$ & $1,05 \pm 0,01$ & $29,52 \pm 0,91$ \\
\hline Eucalipto & $1,18 \pm 0,16$ & $1,09 \pm 0,03$ & $25,84 \pm 4,25$ \\
\hline Cutieira & $0,73 \pm 0,05$ & $1,11 \pm 0,03$ & $16,15 \pm 0,77$ \\
\hline Pivô & $1,15 \pm 0,11$ & $1,14 \pm 0,01$ & $26,00 \pm 2,35$ \\
\hline Pasto & $0,65 \pm 0,06$ & $1,05 \pm 0,01$ & $13,63 \pm 1,02$ \\
\hline
\end{tabular}

$\mathrm{C}=$ teor de carbono do solo (Walkey \& Black); $\mathrm{DS}=$ densidade do solo; $\mathrm{EC}=$ Estoque de carbono do solo. 
al e, na camada subsuperficial, Pasto < Cutieira < Pivô < Eucalipto < Mata. A fração AH variou de 1,39 a 2,59 g $\mathrm{kg}^{-1}$ e de 0,47 a $1,68 \mathrm{~g} \mathrm{~kg}^{-1}$ nas camadas superficial e subsuperficial, respectivamente.

Os valores da fração Huminas tenderam a serem superiores nos sistemas Florestais, em comparação com os de gramíneas (Pasto e Pivô). As amplitudes foram 3,60 a 7,53 $\mathrm{g} \mathrm{kg}^{-1}$ na camada 0 a $20 \mathrm{~cm}$ e 1,90 a 7,26 na camada 20 a $40 \mathrm{~cm}$.

Dentre as frações, em média, MOL, AFL, AF, AH e Huminas representaram, respectivamente, 13, 14, 14, 16 e $43 \%$ do $\mathrm{C}_{\mathrm{T}}$, na camada superficial, e $8,16,14,13$ e $53 \%$ do $\mathrm{C}_{\mathrm{T}}$, na camada subsuperficial.

As somas das frações, que representam uma taxa de recuperação, em cada sistema em estudo, para ambas as profundidades, variou de 95 a $108 \%$ do carbono total. De forma geral, as frações MOL, AFL e AF são as mais hidrofílicas e alifáticas, portanto, tendem a ser mais solúveis e móveis nos sistemas naturais.

A relação entre AH e AF das amostras superficiais somente não foi superior à da unidade no sistema Pivô. Por outro lado, na camada subsuperficial, somente assumiu valores maiores que 1,0 na área de Mata. Relações entre AH e AF menores que 1,0 indicam predomínio de formas mais solúveis, hidrofílicas e alifáticas. Por outro lado, são indicadoras da maior presença de $\mathrm{AH}$, indicando menor solubilidade (menor tendência de lixiviação), uma vez que é uma fração mais hidrofóbica e aromática. Além disso, revela menores perturbações, pois os AH originam-se da persistência das formas mais solúveis no sistema, ou seja, de ambiência propícia em termos de disponibilidade de substrato e de atividade biológica, para resultar num balanço positivo entre as perdas por erosão e por lixiviação e o incremento do estoque de carbono, em formas mais humificadas
(Baldotto et al., 2010). Essas condições foram mais adequadas para a humificação, na área de Mata, seguida pelos sistemas integrando florestas à pastagem, do que nos sistemas de monocultura, mostrando que, além de maiores estoques, os sistemas envolvendo florestas promovem maior estabilidade do carbono sequestrado.

A análise multivariada de todo o conjunto dos dados gerou os dendrogramas das Figuras 1 e 2, os quais indicaram a divisão em grupos de amostras, com maior ou menor distancia euclidiana, ou seja, menos ou mais semelhantes (Cruz, 2006), em ambas as profundidades de amostragem.

Nessa divisão, para as amostras superficiais, os sistemas envolvendo espécies florestais (Mata, Eucalipto e Cutieira) agruparam-se separadamente dos solos sob cultivo ou monocultura de pastagem (Pivô e Pasto). Ainda, no dendrograma, para a camada 0 a $20 \mathrm{~cm}$, observase que a Mata destaca-se em seu subgrupo, neste, sendo dissimilar. Já na camada subsuperficial, Mata e Pasto são mais semelhantes entre si, enquanto Cutieira, Pivô e Eucalipto aparecem em conjunto no dendrograma. Pode também ser observado que as áreas de Pivô e de Cutieira formam um subgrupo separado da área de Eucalipto.

Pode-se observar que os valores de matéria orgânica são superiores, na Mata e no Eucalipto, mostrando que sistemas florestais produzem uma serrapilheira maior, que resulta em estoque de carbono mais expressivo (Miranda et al., 2007). Os valores de acidez estão inversamente relacionados com os da saturação por bases. Os valores excessivos de acidez no sistema Eucalipto mostram uma acidificação progressiva, em comparação com os do sistema Mata e, também, com os dos demais manejos estudados. Uma possível explicação para os mais baixos níveis de bases trocáveis, nos dois sistemas florestais de Eucalipto e Cutieira, pode

Tabela 3. Fracionamento do carbono orgânico do solo sob manejo florestal, agrícola e pastoril

\begin{tabular}{|c|c|c|c|c|c|c|c|c|c|}
\hline \multirow{3}{*}{ Amostra } & \multirow{3}{*}{ Camada } & \multicolumn{8}{|c|}{ Fracionamento $^{(1)}$} \\
\hline & & MOL & AFL & $\mathbf{A F}$ & $\mathbf{A H}$ & HUM & CT & REC & AH/AF \\
\hline & & \multicolumn{6}{|c|}{$\mathrm{g} \mathrm{kg}^{-1}$} & $\%$ & \\
\hline \multirow{2}{*}{ Mata } & 0 a 20 & $2,20 \pm 0,11$ & $1,98 \pm 0,08$ & $2,13 \pm 0,22$ & $2,59 \pm 0,02$ & $6,69 \pm 0,09$ & $15,70 \pm 0,19$ & $99 \pm 3$ & $1,23 \pm 0,12$ \\
\hline & 20 a 40 & $0,87 \pm 0,02$ & $1,68 \pm 0,07$ & $1,60 \pm 0,14$ & $1,68 \pm 0,38$ & $7,26 \pm 0,28$ & $12,67 \pm 0,19$ & $103 \pm 1$ & $1,08 \pm 0,34$ \\
\hline \multirow{2}{*}{ Eucalipto } & 0 a 20 & $2,67 \pm 0,31$ & $2,47 \pm 0,13$ & $1,96 \pm 0,06$ & $2,25 \pm 0,32$ & $6,06 \pm 0,18$ & $16,27 \pm 0,38$ & $95 \pm 4$ & $1,15 \pm 0,20$ \\
\hline & 20 a 40 & $0,83 \pm 0,04$ & $2,35 \pm 0,15$ & $1,47 \pm 0,15$ & $1,36 \pm 0,62$ & $4,94 \pm 0,18$ & $10,02 \pm 0,19$ & $109 \pm 6$ & $0,98 \pm 0,52$ \\
\hline \multirow{2}{*}{ Cutieira } & 0 a 20 & $1,63 \pm 0,07$ & $1,23 \pm 0,08$ & $1,89 \pm 0,16$ & $2,08 \pm 0,11$ & $7,53 \pm 0,10$ & $14,56 \pm 0,19$ & $99 \pm 5$ & $1,11 \pm 0,04$ \\
\hline & 20 a 40 & $0,60 \pm 0,02$ & $0,94 \pm 0,07$ & $0,94 \pm 0,22$ & $0,81 \pm 0,33$ & $2,55 \pm 0,29$ & $5,48 \pm 0,19$ & $106 \pm 5$ & $0,99 \pm 0,58$ \\
\hline \multirow{2}{*}{ Pivô } & 0 a 20 & $1,19 \pm 0,07$ & $1,52 \pm 0,08$ & $1,52 \pm 0,07$ & $1,39 \pm 0,19$ & $3,60 \pm 0,19$ & $8,51 \pm 0,19$ & $108 \pm 1$ & $0,92 \pm 0,17$ \\
\hline & 20 a 40 & $0,50 \pm 0,02$ & $0,65 \pm 0,07$ & $1,23 \pm 0,07$ & $1,22 \pm 0,27$ & $5,48 \pm 0,19$ & $8,89 \pm 0,89$ & $102 \pm 9$ & $0,98 \pm 0,17$ \\
\hline \multirow{2}{*}{ Pastagem } & 0 a 20 & $1,25 \pm 0,01$ & $1,83 \pm 0,07$ & $1,83 \pm 0,08$ & $2,06 \pm 0,28$ & $4,94 \pm 0,20$ & $11,54 \pm 0,19$ & $103 \pm 2$ & $1,12 \pm 0,11$ \\
\hline & 20 a 40 & $0,59 \pm 0,04$ & $0,95 \pm 0,07$ & $0,59 \pm 0,00$ & $0,47 \pm 0,12$ & $1,90 \pm 0,19$ & $4,35 \pm 0,19$ & $104 \pm 2$ & $0,81 \pm 0,20$ \\
\hline
\end{tabular}

(1) Fracionamento: $\mathrm{MOL}=$ matéria orgânica leve; $\mathrm{AFL}=$ ácidos fúlvicos livres; $\mathrm{AF}=$ ácidos fúlvicos; $\mathrm{HUM}=$ huminas; $\mathrm{CT}=$ carbono total; $\mathrm{REC}=$ recuperação do método em relação ao CT; $\mathrm{AH} / \mathrm{AF}$ = relação entre as frações ácidos húmicos e ácidos fúlvcos. 
ser a de que os nutrientes que estavam inicialmente no solo encontrem-se atualmente, na biomassa das árvores. Além disso, os sistemas mais ricos em carbono e base, também apresentaram mais alta CTC. As formas de carbono do solo contribuem para a troca iônica, por meio da geração de cargas elétricas em grupos carboxílicos e fenólicos da matéria orgânica, sendo os principais constituintes da CTC de solos tropicais (Baldotto et al., 2008). O sistema Mata, em média, apresentou uma maior fertilidade, em comparação com a do sistema Eucalipto, considerando-se a capacidade de troca catiônica efetiva, a soma e a saturação por bases (Alvarez et al, 1999; Novais et al., 2007; Baldotto et al., 2010). Os horizontes superficiais dos sistemas analisados apresentam baixas concentrações de $\mathrm{P}$ e baixa ferti- lidade. A menor saturação por bases nas duas camadas amostradas foi apresentada pelo sistema Eucalipto, condizente com os mais altos valores de saturação por alumínio (m \%). Os resultados de fertilidade estão associados aos dos estoques de carbono, sendo os valores dos sistemas florestais superiores aos dos sistemas com maiores grau de alteração (Pastagem e Pivô).

Com os resultados, pode-se inferir que o sistema remanescente da mata nativa tendeu a manter os estoques de carbono e a fertilidade natural, ou, pelo menos, esta pode ter diminuído menos em relação àquela original, pois foi superior à dos sistemas atuais de uso. Contudo, os sistemas envolvendo a integração de florestas de Eucalipto e Cutieira nas antigas pastagens apresentaram dados muito próximos aos da área de Mata e superi-

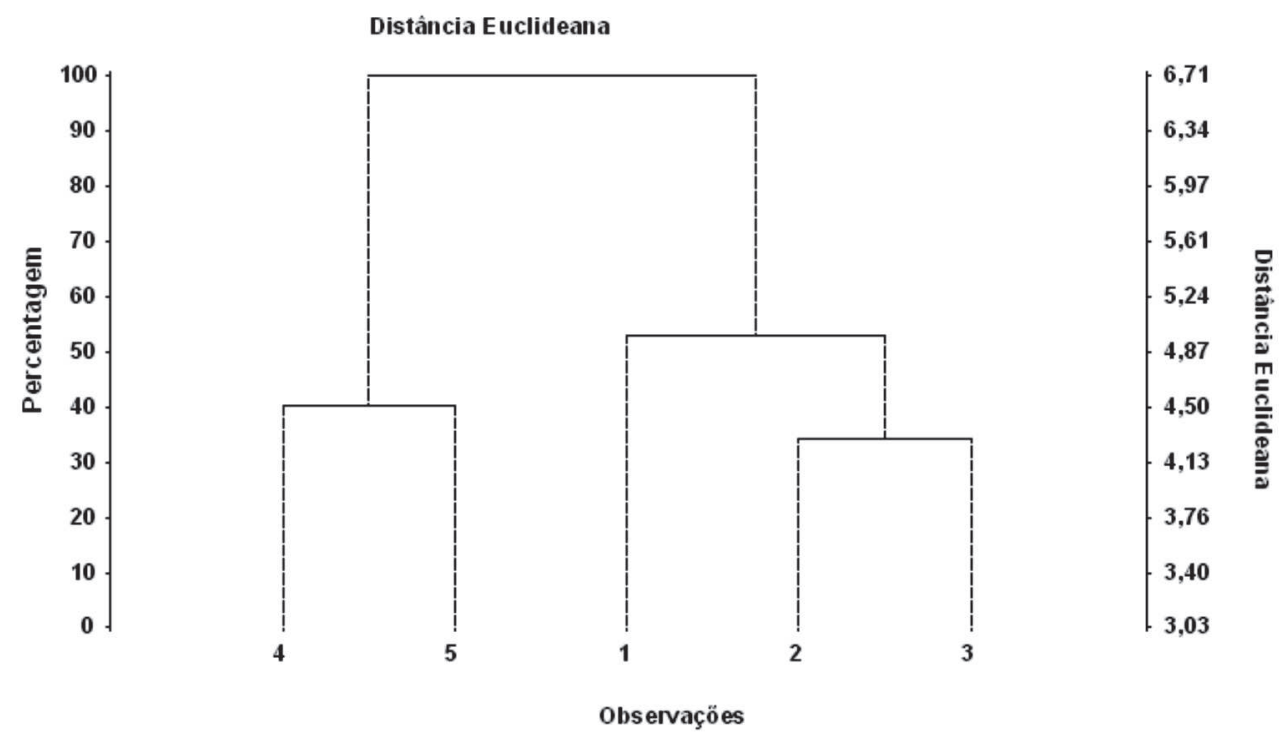

Figura 1. Agrupamento pela variância mínima e distância euclidiana usando-se a análise multivariada para o conjunto de dados das amostras estudadas, na camada 0 a $20 \mathrm{~cm}$ (1= Mata, 2=Eucalipto; 3=Cutieira; 4=Pivô e 5=Pasto).

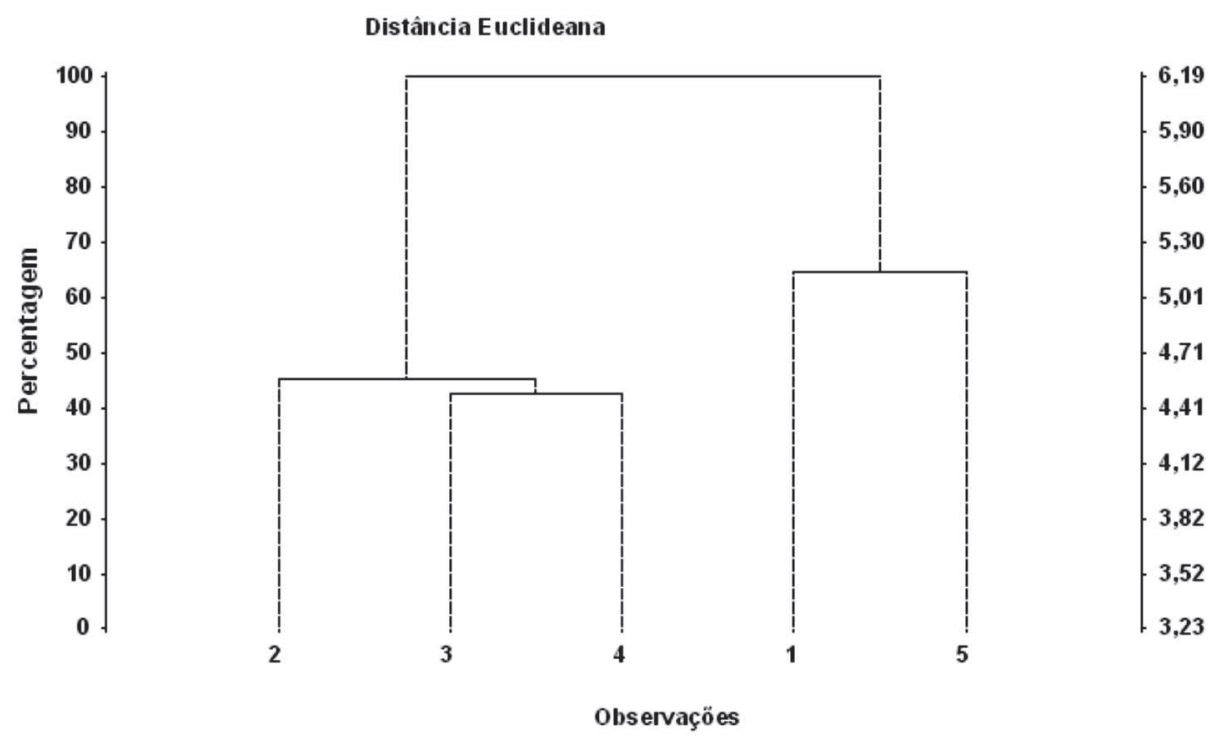

Figura 2. Agrupamento pela variância mínima e distância euclidiana usando-se a análise multivariada para o conjunto de dados das amostras estudadas, na camada 20 a 40 cm (1= Mata, 2=Eucalipto; 3=Cutieira; 4=Pivô e 5=Pasto). 
ores aos dos solos continuamente cultivados ou com monocultura de pastagem. Os resultados de Dixon et al. (1994), Feixo et al. (2002), Araújo et al. (2007), Miranda et al. (2007), Ribas et al. (2008) seguiram tendências semelhantes, em diferentes biomas brasileiros.

No sistema Mata, foram também verificados os mais altos valores de aromaticidade e de hidrofobicidade da matéria orgânica do solo (maior relação AH/AF), indicando superior estabilidade dos estoques de carbono (Rice \& Maccarthy, 1991; Stevenson, 1994; Zech et al., 1997; Baldotto et al., 2010), ou seja, a floresta apresenta maior quantidade e estabilidade dos estoques de carbono do solo (persistência do "sequestro" de C).

$\mathrm{O}$ acúmulo e armazenamento da matéria orgânica do solo (MOS) é um dos atributos do solo mais fortemente relacionados com a sua qualidade, tendo sido utilizado como indicador, especialmente por ser altamente variável com o sistema de manejo adotado e correlacionarse com a maioria dos atributos relacionados com a qualidade do solo (Stevenson, 1994; Camargo et al., 2008). Com o aumento dos teores de MOS e a ativação de diversos processos químicos, físicos e biológicos no solo, manifestam-se propriedades como maiores resistência à erosão, taxa de infiltração e retenção de água no solo, capacidade de retenção de cátions, estoques e complexação de $\mathrm{Al}^{3+}$ e ciclagem de nutrientes e outros elementos químicos, possibilitando maior resistência a perturbações, ou seja, conferindo maior resiliência e elevada fertilidade (Cerri et al., 2003; Conceição et al., 2005).

Infere-se, assim, que a perda de fertilidade, ao se remover a vegetação natural, esteja associada à maior tendência a perdas de nutrientes por erosão e lixiviação, o que pode provocar, ainda, tendência ao assoreamento e à eutrofização dos sistemas aquáticos adjacentes. Mesmo considerando-se todas as análises químicas deste trabalho, é difícil a conceituação de fertilidade do solo, neste novo milênio (Denardin \& Kochhann, 2007). No entanto, os indicadores de fertilidade (Tabela 1), sobretudo das variáveis que avaliam a disponibilidade de bases, a adsorção de $\mathrm{P}$, a acidez e a retenção de nutrientes aumentam com os estoques de carbono do solo, como foi também observado por Carvalho et al. (2010) e por Baldotto et al. (2010). Assim, a diminuição dos estoques de carbono é acompanhada pela diminuição da fertilidade do solo.

Cerri et al. (2003) observou que a intervenção antrópica nos ecossistemas naturais, representada pela conversão da floresta nativa em pastagem cultivada e submissão da pastagem nativa ao sistema de pastejo contínuo, promoveu significativa redução dos estoques de $C$, dado o menor aporte de resíduos orgânicos às pastagens, determinado pela marcante extração de biomassa aérea desses ecossistemas, imposta pela pressão de pastejo.
Ribas et al. (2008), na Região dos Lagos fluminense, mostrou resultados que apontam que o fracionamento químico da matéria orgânica do solo e o seu potencial de lixiviação para o sistema aquático modificaram-se, conforme o sistema solo-cobertura estudado. Os autores relataram que a mobilidade diferencial, a concentração e a qualidade da matéria orgânica, em sistemas lacustres, estão relacionadas com o uso do solo no entorno, afetando as relações ecológicas. Os dados chamam atenção para a dificuldade de indicadores para o monitoramento, apresentando a quantidade e a qualidade das formas de carbono orgânico como as variáveis mais sensíveis.

No trabalho de Ribas et al. (2008), dentre os sistemas estudados (pastagem, agricultura, cana e mata nativa), o sistema florestal apresentou maior equilíbrio entre as formas de carbono, com maior estabilidade da matéria orgânica do solo. A mobilidade do carbono variou de acordo com a distribuição das frações da matéria orgânica do solo. Sistemas em que predominaram formas mais solúveis e menos humificadas apresentaram maior potencial de lixiviação. Neste trabalho, observaram-se dados semelhantes e, dessa forma, pode-se inferir que os sistemas florestais, principalmente a Mata, tendem a perder menos carbono por lixiviação para o sistema aquático. Essa lixiviação de carbono, se excessiva, entre outros fatores, pode aumentar a demanda biológica por oxigênio, trazendo complicações para a disponibilidade de oxigênio molecular para a respiração de peixes e outros seres aquáticos (Stumm \& Morgan, 1996; Baird \& Cann, 2011).

Destaca-se a maior densidade do solo revelada pela amostra Pivô, devida a práticas tradicionais agrícolas, como aeração e gradeamento. O aumento do grau de compactação do solo acarreta a diminuição de poros importantes para os fluxos de gases e a solução do solo, além de efeitos na difusão de nutrientes, na resistência à penetração etc (Anjos et al., 2007; Novais et al., 2007; Figueiredo et al., 2008).

Os sistemas de manejo do solo integrando florestas à monocultura mostraram-se de fundamental importância para a manutenção da quantidade e da qualidade da matéria orgânica e da fertilidade do solo e, portanto, permitem concluir que a adoção de práticas de manejo adequadas é de grande relevância para a sustentabilidade dos sistemas agropecuários e florestais. Dos pontos de vista agronômico e geoquímico ambiental, os maiores e mais estáveis estoques de carbono dos sistemas florestais confirmam que essas formas de manejo são mais adequadas que o cultivo contínuo ou a monocultura de forragem, fortalecendo a ideia de integração de lavoura, pecuária e floresta, como prática de manejo mais sustentável. 


\section{CONCLUSÕES}

Os sistemas florestais, principalmente a Mata, em comparação com o cultivo contínuo ou a monocultura de pastagem, apresentaram maior fertilidade do solo, maiores estoques de carbono mais estáveis e menos solúveis, com formas mais aromáticas e hidrofóbicas (maior relação $\mathrm{AH} / \mathrm{AF})$.

A quantificação dos estoques de carbono, associada às estimativas da sua estabilidade, fornece indicadores estreitamente relacionados com a fertilidade do solo, possibilitando a avaliação de práticas anteriores e o monitoramento de práticas presentes e futuras, em ecossistemas naturais e agrários, e o desenvolvimento de estratégias para o seu manejo e conservação.

\section{REFERÊNCIAS}

Alvarez V VH, Novais RF, Barros NF, Cantarutti RB \& Lopes AS (1999) Interpretação dos resultados das análises de solos. In: Ribeiro AC, Guimarães PTG \& Venegas VHA(Eds.) Recomendações para uso de corretivos e fertilizantes em Minas Gerais: $5^{\mathrm{a}}$ Aproximação. Viçosa, Comissão de Fertilidade do Solo do Estado de Minas Gerais. p.25-32

Anjos JT, Uberti AAA, Vizzoto VJ, Leite GB \& Kriegrer M (1994) Propriedades físicas do solo sob diferentes sistemas e usos de manejo. Revista Brasileira de Ciência do Solo, Campinas, 18:139-145.

Araújo R, Goedert WJ \& Lacerda MPC (2007) Qualidade de um solo sob diferentes usos e sob cerrado nativo. Revista Brasileira de Ciência do Solo, 31:1099-1108.

Baird C \& Cann M (2011) Química Ambiental. 4ª ed. Porto Alegre, Bookman. 844p.

Baldotto MA, Canellas LP, Mendonca ES, Dobbss LB, Velloso ACX \& Amaral Sobrinho NMB (2008) Reações da matéria orgânica In: Santos G \& Camargo F (Eds.) Fundamentos da matéria orgânica do solo: ecossistemas tropicais e subtropicais. $2^{\mathrm{a}}$ ed. Porto Alegre, Metrópole. p. $45-63$.

Baldotto MA, Canela MC, Canellas LP, Dobbss LB \& Velloso ACX (2010) Redox índex of soil carbon stability. Revista Brasileira de Ciências do Solo, 34:1543-1551.

Carvalho JLN, Avanzi JC, Silva MLN, Mello CR \& Cerri CEP (2010) Potencial de sequestro de carbono em diferentes biomas do Brasil Revisão de Literatura. Revista Brasileira de Ciências do Solo, 34:277289.

Cerri CEP, Coleman K, Jenkinson DS, Bernoux M, Victoria R \& Cerri CC (2003) Modeling soil carbon from forest and pasture ecosystems of Amazon, Brazil. Soil Science Society of America Journal, 67:879-1887.

Cibapar (2013) Consórcio Intermunicipal da Bacia Hidrográfica do Rio Paraopeba. Disponível em: <http://www.aguasdoparaopeba.org.br/ index.php>. Acessado em: 21 de março de 2013.

Coelho MR, Santos HG, Silva EF \& Aglio MLD (2002) O recurso natural solo. In: Manzatto CV, Freitas JR \& Peres JRR (Eds.) Uso agrícola dos solos brasileiros. Rio de Janeiro, Embrapa Solos. p. 01-11.

Conceição PC, Amado TJC, Mielniczuk J \& Spagnollo E (2005) Qualidade do solo em sistemas de manejo avaliada pela dinâmica da matéria orgânica e atributos relacionados. Revista Brasileira de Ciência do Solo, 29:777-788.

Correia JR, Reatto A \& Spera ST (2004) Solos e suas relações com o uso e o manejo. In: Sousa DMG \& Lobato E (Eds.) Cerrado: correção do solo e adubação. $2^{a}$ ed. Brasília, Embrapa. p. 29-58.
Cruz CD (2006) Análise multivariada e simulação. Viçosa, Editora UFV. $175 \mathrm{p}$.

Denardin JE \& Kochhann RA (2007) Desafios à caracterização de solo fértil em manejo e conservação do solo e da água. Revista Plantio Direto, 98:16-21.

Derpsch R (1997) Agricultura sustentável. In: Saturnino HM \& Landers JN (Eds.) O meio ambiente e o plantio direto. $1^{\mathrm{a}}$ ed. Goiânia, EmbrapaSPI. p. $29-48$.

Dias LE \& Griffith JJ (1998) Conceituação e caracterização de áreas degradadas. In: Dias LE \& Mello JWV (Eds.) Recuperação de áreas degradadas. Viçosa, UFV. p. 01-07.

Embrapa - Empresa Brasileira de Pesquisa Agropecuária (2006) Sistema Brasileiro de Classificação de Solos. $2^{\mathrm{a}}$ ed. Rio de Janeiro, Centro Nacional de Pesquisa em Solos. 412p.

Embrapa - Empresa Brasileira de Pesquisa Agropecuária (1997) Manual de métodos de análise de solo $2^{\mathrm{a}}$ ed. Rio de Janeiro, Centro Nacional de Pesquisa em Solos. 212p.

Eswaran H, van den Berg E \& Reich P (1993) Organic carbon in soils of the word. Soil Science Society American Journal, 57:192-194.

Fang C \& Moncrieff JB (2001) The dependence of soil $\mathrm{CO}_{2}$ efflux on temperature. Soil Biology and Biochemistry, 33:155-165.

IPCC - Intergovernmental Panel on Climate Change (2001) Climate change: The scientific basis. Cambridge, Cambridge University Press. $881 \mathrm{p}$.

Lal R (2004) Soil carbon sequestration impacts on global climate change and food security. Science, 304:1623-1627.

Reis TC \& Rodella AA (2002) Cinética de degradação da matéria orgânica e variação do $\mathrm{pH}$ do solo sob diferentes temperaturas. Revista Brasileira de Ciência do Solo, 26:619-626.

Ribas LM, Baldotto MA, Canellas L P \& Rezende CE (2008) Qualidade e mobilidade da matéria orgânica de sistemas adjacentes à Lagoa de Cima, Campos dos Goytacazes. Geochimica Brasiliensis, 22:103-112.

Sposito G (2008) The chemistry of soils, $2^{\text {nd }}$ ed. New York, Oxford University Press. 330p.

Steel RGD \& Torrie JH (1980) Principles and procedures of statistics. $2^{\text {nd }}$ ed. New York, McGraw-Hill. 633p.

Stevenson FJ (1994) Humus chemistry: genesis, composition, reactions. $2^{\text {nd }}$ ed. New York, Wiley. 496p.

Stumm W \& Morgan JJ (1996) Aquatic chemistry: chemical equilibria and rates in natural waters. $3^{\text {nd }}$ ed. New York, Wiley. $1022 \mathrm{p}$.

Rice JA \& Maccarthy P (1991) Statistical evaluation of the elemental composition of humic substances. Organic Geochemistry, 17:635648.

Zech W, Senesi N, Guggenberger G, Kaiser K, Lehmann J, Miano TM, Miltner A \& Schroth G (1997) Factors controlling humification and mineralization of soil organic matter in the tropics. Geoderma, 79:117161.

Thurman EM \& Malcolm RL (1981) Preparative isolation of aquatic humic substances. Environmental Science \& Technology, 15:463466. 